\title{
Searching for the QCD critical point via the rapidity dependence of cumulants
}

\author{
Jasmine Brewer, ${ }^{1}$ Swagato Mukherjee, ${ }^{2}$ Krishna Rajagopal, ${ }^{1}$ and Yi Yin ${ }^{1}$ \\ ${ }^{1}$ Center for Theoretical Physics, Massachusetts Institute of Technology, Cambridge, Massachusetts 02139, USA \\ ${ }^{2}$ Physics Department, Brookhaven National Laboratory, Upton, New York 11973, USA
}

(Received 18 May 2018; published 13 December 2018)

\begin{abstract}
The search for a possible critical point in the QCD phase diagram is ongoing in heavy-ion collision experiments at the BNL Relativistic Heavy Ion Collider (RHIC) which scan the phase diagram by scanning the beam energy; a coming upgrade will increase the luminosity and extend the rapidity acceptance of the solenoidal tracker at RHIC (STAR) detector. In fireballs produced in RHIC collisions, the baryon density depends on rapidity. By employing Ising universality together with a phenomenologically motivated freeze-out prescription, we show that the resulting rapidity dependence of cumulant observables sensitive to critical fluctuations is distinctive. The dependence of the kurtosis (of the event-by-event distribution of the number of protons) on rapidity near midrapidity will change qualitatively if a critical point is passed in the scan. Hence, measuring the rapidity dependence of cumulant observables can enhance the prospect of discovering a critical point, in particular if it lies between two energies in the beam energy scan.
\end{abstract}

DOI: 10.1103/PhysRevC.98.061901

A central goal of heavy-ion collision experiments is to map the QCD phase diagram as a function of temperature $T$ and baryon chemical potential $\mu_{B}$ [1-3]. At zero $\mu_{B}$, the phase diagram features a continuous crossover from quark-gluon plasma (QGP) to ordinary hadronic matter as a function of decreasing $T$ [4-8]. Increasing $\mu_{B}$ corresponds to doping the QGP with an excess of quarks over antiquarks. As in condensed-matter physics, after the discovery of a new form of strongly correlated matter, here the strongly coupled liquid called QGP, a more complete understanding requires mapping its phase diagram as a function of $T$ and doping. It is an open question whether the crossover at zero doping becomes a sharp first-order phase transition as the doping $\mu_{B}$ is increased beyond some critical point [3,9]. At nonzero $\mu_{B}$ where lattice calculations become extremely difficult $[10,11]$, there are no first-principles theoretical calculations which provide reliable guidance as to whether there is a critical point in the phase diagram of QCD, or its location if it does exist [12-15]. Model calculations suggest the existence of a critical point, but disagree wildly on its location in the $\left(\mu_{B}, T\right)$ plane $[14,15]$. Reducing the beam energy increases the $\mu_{B}$ of the QGP produced in a heavy-ion collision [3,16-18] (principally because lower energy collisions make less entropy but also because they deposit more of their baryon number in the plasma) but it also reduces the temperatures achieved. So, these experiments can scan the crossover (and potentially

Published by the American Physical Society under the terms of the Creative Commons Attribution 4.0 International license. Further distribution of this work must maintain attribution to the author(s) and the published article's title, journal citation, and DOI. Funded by SCOAP . critical) regime of the phase diagram out to some value of $\mu_{B}$ corresponding to the lowest energy collisions that reach the crossover (critical) temperature [1,2]. If a critical point is located in the regime that is within reach, it may be detected experimentally.

The search for a critical point in the phase diagram of QCD at the BNL Relativistic Heavy Ion Collider (RHIC) is currently underway, with collisions at energies ranging from $\sqrt{s}=200 \mathrm{AGeV}$ down to $\sqrt{s}=7.7 \mathrm{AGeV}$, producing fireballs that freeze-out with chemical potentials in the range $25 \lesssim \mu_{B} \lesssim 400 \mathrm{MeV}[17,18]$. This exploration will be extended to higher $\mu_{B}$, albeit at lower temperatures, via collisions at lower $\sqrt{s}$ at the Nuclotron-based Ion Collider fAcility (NICA) at the Joint Institute for Nuclear Research (JINR) in Dubna, Russia [19], now under construction, and fixed target collisions at RHIC and at the future GSI Facility for Antiproton and Ion Research (FAIR) [20]. Phase I of the RHIC beam energy scan (BES-I) was completed in 2014, with no signs of a critical point for $\mu_{B}<200 \mathrm{MeV}$ and with tantalizing but inconclusive results at larger $\mu_{B}$, in collisions with $19.6 \geqslant \sqrt{s} \geqslant 7.7 \mathrm{AGeV}[1,2,9,21-23]$. Phase 2 of the scan (BES-II), to begin in 2019 [9,23], will focus on this regime with increased luminosity and consequently much higher statistics. One of the improvements planned before BES-II is an upgrade of the inner time projection chamber (iTPC) at STAR, which will extend its rapidity acceptance for protons from $|y|<0.5$ in BES-I to $|y|<0.8$ in BES-II [24].

The energy of a heavy-ion collision sets the initial $T$ and $\mu_{B}$ of the QGP which is created, with lower energy collisions being more baryon rich. The QGP then follows a trajectory in the $\left(T, \mu_{B}\right)$ plane as it expands and cools. If there is a critical point in the QCD phase diagram within the range of $\mu_{B}$ which is accessible in the BES, then at some collision energies the fireball produced may pass through or near the critical region, while at higher (lower) collision energies the 
fireball produced will pass the critical point on the low (high) $\mu_{B}$ side. The theoretical challenge is to describe the unique signatures of this scenario which would be observable in data from the BES.

A critical point in a thermodynamic system is characterized by an enhanced correlation length. Although the correlation length itself is not observable because the critical order parameter $\sigma$ couples to all hadrons, the $n$th cumulant moments $\kappa_{n}$ of the event-by-event distribution of the measured multiplicity $N$ of various particle species, for example, $\kappa_{4}[N]=$ $\left\langle(\delta N)^{4}\right\rangle-3\left\langle(\delta N)^{2}\right\rangle^{2}$, scale with powers of the correlation length $\xi$ near a critical point [25]. Protons couple more strongly to $\sigma$ than pions or kaons, making cumulants of the proton multiplicity good observables with which to look for critical fluctuations [26,27]. The non-Gaussian cumulants $\kappa_{3}[N]=\left\langle(\delta N)^{3}\right\rangle \sim \xi^{9 / 2}$ and $\kappa_{4}[N] \sim \xi^{7}$ scale with higher powers of the correlation length than the Gaussian cumulants and are therefore more sensitive to critical behavior [25,27]. Furthermore, an analysis that is valid for any critical point in the same (3D Ising) universality class has shown that $\kappa_{4}$ will also change sign near a QCD critical point [28,29]. Nonmonotonic behavior and a sign change of the fourth cumulant as a function of the beam energy are characteristic signatures of the presence of a critical point which can be searched for in the RHIC BES.

The dependence of fluctuation measures on the total rapidity acceptance has been studied before [30-32] upon assuming that $\mu_{B}$, and hence $\xi$ and $\kappa_{4}$, do not depend on rapidity. However, the baryon density does depend nontrivially on rapidity at RHIC BES energies (see, e.g., Refs. [33-35]). Even at top RHIC and LHC energies, the baryon density is significant at very large rapidity, around two units of rapidity below that of the incident beams, although building instrumentation to make the required measurements to explore the hot baryon-rich matter produced in this regime presents considerable challenges $[3,33]$. Since the correlation length and fluctuations become very large near a critical point, the rapidity dependence of the baryon density gives rise to a strong, nontrivial rapidity dependence of the cumulants near a critical point which was not incorporated in previous work. Furthermore, since each rapidity is associated with a different value of $\mu_{B}$ and therefore probes a different part of the critical region on the phase diagram, we shall see that integrating over the full rapidity acceptance averages out interesting features in the cumulants which are characteristic of critical behavior. Instead, we propose that binning the cumulants in rapidity gives a more crisp picture of the critical regime, and demonstrate that the rapidity dependence of these binned cumulants near midrapidity will change qualitatively if a critical point is passed in the BES. We therefore propose this observable as a complementary means by which to observe the presence of a critical point at the BES. In particular, it provides a new, and distinctive, signature by which to determine whether downward steps in the collision energy take us past a critical point in the phase diagram.

Rapidity dependence of $\mu_{B}$. In this Rapid Communication, we illustrate the effect that the rapidity dependence of $\mu_{B}$ at RHIC BES energies has on the rapidity dependence of cumulants, and propose using this toward discovering (or ruling out) a critical point using RHIC BES data.

Since the baryon density at freeze-out is symmetric in the space-time rapidity $y_{s}$ for symmetric heavy-ion collisions, for small $\left|y_{s}\right|$ the deviation from boost invariance takes the form

$$
\mu_{B}\left(y_{s}\right) \sim \mu_{B, 0}+\alpha y_{s}^{2},
$$

with $\mu_{B, 0}$ and $\alpha$ constants that depend on the beam energy $\sqrt{s}$. We shall use this form for illustrative purposes, noting of course that it cannot be relied upon at large $\left|y_{s}\right|$. As we have discussed, the basis of the BES is that downward steps in $\sqrt{s}$ yield upward steps in $\mu_{B, 0}$. For illustrative purposes, we shall pick three values of $\mu_{B, 0}$ within the BES range and see what happens if these steps were to happen to take us past a possible critical point. The value of $\alpha$ has been measured in CERN Super Proton Synchrotron (SPS) collisions with $\sqrt{s}=17.3 \mathrm{AGeV}$, where $\alpha=50 \mathrm{MeV}$ [34]. At this (and all higher, and some lower) beam energies, $\alpha>0$ because the baryon number density is peaked at roughly two units of rapidity below the beam rapidity, meaning that it is less at $y_{s}=0$ than at larger $\left|y_{s}\right|[3,16]$. In BNL Alternating Gradient Synchrotron (AGS) collisions with $\sqrt{s}=5.5 \mathrm{AGeV}$, though, the beam rapidity is low enough that the baryon number density peaks at $y_{s}=0$ and $\alpha<0$ [16]. The $\sqrt{s}$ at which $\alpha$ changes from positive to negative is not known but is likely near the lower end of the BES range. Ultimately, measurements of the ratios of the mean particle number distribution for different species from the RHIC BES should be used to measure how $\mu_{B}$ at freeze-out depends on $y_{s}$ at each BES collision energy, and hence to determine the value of $\alpha$ at each energy. For illustrative purposes here, we shall investigate the consequences of choosing $\alpha=50 \mathrm{MeV}$ at each of our three values of $\mu_{B, 0}$ as well as checking how things change if we choose $\alpha=-50 \mathrm{MeV}$ instead at our largest value of $\mu_{B, 0}$.

Cumulants in the critical regime. Order parameter fluctuations near a critical point induce fluctuations in the event-byevent particle multiplicities. Throughout this work, we will consider the cumulants of protons, as these are expected to be most sensitive to critical fluctuations [27]. From previous work [25,27,28,31], the contribution to the fourth cumulant of the proton multiplicity distribution coming from critical fluctuations (denoted by the subscript $\sigma$ ) takes the form

$$
\kappa_{4}[N]_{\sigma}=\int_{\mathbf{x}} K_{4} \xi^{7} T^{2}\left(g \int_{\mathbf{p}} \frac{\chi_{\mathbf{p}}}{\gamma_{\mathbf{p}}}\right)^{4},
$$

where the $\mathbf{x}$ integral is a space-time integral over the freezeout hypersurface, where $T, \mu_{B}$, and consequently $\xi\left(\mu_{B}, T\right)$ and $K_{4}\left(\mu_{B}, T\right)$ (proportional to the kurtosis of the eventby-event distribution of the fluctuating order parameter, see below) can take on different values at different points on the freeze-out hypersurface, where $g$ is the $\sigma$-proton-proton coupling which we set to the same benchmark value $g=7$ used in Ref. [27], where the $\mathbf{p}$ integral is a momentumspace integral over the protons at the point $\mathbf{x}$, where $\chi_{\mathbf{p}}=$ $f_{\mathbf{p}}\left(1-f_{\mathbf{p}}\right) / T$ if we assume local equilibrium with $f_{\mathbf{p}}$ the Fermi-Dirac distribution boosted by the radial flow velocity at the point $\mathbf{x}$, and where $\gamma_{\mathbf{p}}=\sqrt{\mathbf{p}^{2}+m^{2}} / m$ with $m$ the proton mass. We note that this freeze-out prescription allows 
us to convert the space-time rapidity dependence of $\mu_{B}$ into momentum-space rapidity dependence of $\kappa_{4}[N]_{\sigma}$. [See Eq. (3) for details.] We follow Ref. [31] and use a blast wave model to obtain the radial flow velocity and freeze-out hypersurface, taking the freeze-out curve in the $\left(T, \mu_{B}\right)$ plane from the fit to experimental data found in Refs. [17,18]. Following Ref. [31], we shall make the approximation $\chi_{\mathbf{p}} \approx f_{\mathbf{p}} / T$ and use the Boltzmann distribution for $f_{\mathbf{p}}$, allowing us to do some of the integrals analytically.

The shape of the dependence of $K_{4}$ and $\xi$ on $\mu_{B}$ and $T$ are governed by universal properties of critical fluctuations. A critical point in the QCD phase diagram, if it exists, is known to be in the same universality class as the 3D Ising model [3640]. The mapping of the Ising variables $(r, h)$ onto the QCD variables $\left(\mu_{B}, T\right)$ is not universal, but for illustrative purposes we employ the widely used assumption [41] that the Ising $r$ axis (and hence the line of first-order transitions) is parallel to the QCD $\mu_{B}$ axis, and the Ising $h$ axis is parallel to the QCD $T$ axis. For illustrative purposes, we shall place a hypothetical QCD critical point at $\mu_{B}=260 \mathrm{MeV}, T=160 \mathrm{MeV}$. The 3D Ising universality then determines $K_{4}$ at some point away from the critical point in terms of the direction in which that point lies in the $\left(\mu_{B}, T\right)$ plane, and $\xi$ in terms of this angle, the distance away from the critical point, and one nonuniversal parameter whose choice determines the contour on the phase diagram where $\xi=1 \mathrm{fm}$, as illustrated in Fig. 1. Because once $\xi$ is less than $1 \mathrm{fm}$ the magnitude of $\kappa_{4} \propto \xi^{7}$ is negligible, for simplicity we set $\xi=0$ outside the critical regime. (For details, see Refs. [27,28,42,43].)

Following Ref. [31], we cast the momentum integration in terms of the momentum-space rapidity $y$ and transverse momentum $p_{\perp}$, which are measured in experiment:

$$
\int_{\mathbf{p}} \frac{1}{\gamma_{\mathbf{p}}} \rightarrow \frac{2 m}{(2 \pi)^{3}} \int_{y_{c}-\Delta y / 2}^{y_{c}+\Delta y / 2} d y \int_{p_{\min }}^{p_{\max }} p_{\perp} d p_{\perp} \int_{0}^{2 \pi} d \psi .
$$

We have introduced a finite acceptance in both rapidity and transverse momentum. We will keep $p_{\min }=0.4 \mathrm{GeV}$ and $p_{\max }=2 \mathrm{GeV}$ throughout. We shall compute $\kappa_{4}$ using two different kinds of rapidity cuts, either varying $\Delta y$ with $y_{c}=0$, in which case $|y|<y_{\max } \equiv \Delta y / 2$, or varying $y_{c}$ with fixed bin width $\Delta y$.

To simplify the interpretation of our results, we shall show the critical contribution to the cumulants normalized by the average number of protons, $\omega_{4, \sigma} \equiv \kappa_{4}[N]_{\sigma} /\langle N\rangle$. This cumulant ratio has the advantage that if the background (noncritical) contribution were Poisson distributed it would contribute $\omega_{4, \sigma}=1$, meaning that our results in Figs. 1 and 2 should be interpreted as critical contributions to be added to a background of order 1 .

Results and conclusions. In this section, we demonstrate that the rapidity dependence of $\mu_{B}$ makes the rapidity dependence of cumulants sensitive to critical fluctuations in a way that yields distinctive, qualitative, observable consequences. In Fig. 1 we first compute the dependence of the cumulant ratio $\omega_{4, \sigma}$ on the total rapidity acceptance $y_{\max }$. This dependence was studied previously in Ref. [31] upon assuming that $\mu_{B}$ itself is constant in rapidity; we find striking consequences of the rapidity dependence of $\mu_{B}$. Next, motivated by the expanded rapidity coverage that the STAR iTPC upgrade will bring, we compute $\omega_{4, \sigma}$ for bins in rapidity, something that has not been considered previously. We find that the rapidity dependence of the cumulant ratio is a sensitive and interesting probe of critical behavior.

In Fig. 1 we consider a hypothetical set of scenarios motivated by the possibility that there may be a QCD critical point within the energy range to be explored by the RHIC BES. We imagine a critical point at $\mu_{B}^{c}=260 \mathrm{MeV}$, and in the first three rows of the figure we consider heavy-ion collisions with three decreasing values of the beam energy such that freeze-out at midrapidity occurs at $\mu_{B, 0}=200,230$, and $240 \mathrm{MeV}$. In all three rows, we choose $\alpha=50 \mathrm{MeV}$, corresponding to the measured value from SPS collisions with $\sqrt{s}=17.3 \mathrm{GeV}$ and $\mu_{B, 0}=237 \mathrm{MeV}$. Because a real critical point may lie at larger $\mu_{B}^{c}$ than this, where $\alpha$ may become negative, in the fourth row we flip the sign of $\alpha$. The right column of Fig. 1 shows $\omega_{4, \sigma}$ binned in rapidity bins of width $\Delta y=0.4$ centered around $y= \pm y_{c}$, an observable which to our knowledge has not been considered before. This is a more sensitive observable to the unique features of critical behavior than the dependence on the total rapidity acceptance in the center column because it isolates contributions coming from more similar values of $\mu_{B}$, and the correlation length and other features of the critical regime are sensitive to $\mu_{B}$ near $\mu_{B}^{c}$. We see many interesting qualitative features in the rapidity dependence of $\omega_{4, \sigma}$. For example, if $\mu_{B, 0}$ is in the red region, where $\omega_{4, \sigma}$ is negative and relatively small in magnitude, larger and positive contributions to $\omega_{4, \sigma}$ can be found at larger rapidity. This can be seen in the middle panels of the first and second rows, but it is much more striking in the right panels, indicating the value of binning in rapidity. On the other hand, if $\mu_{B, 0}$ lies in the blue region, in the right column the largest value of $\omega_{4, \sigma}$ is obtained for the bin centered at $y=0$, with $\omega_{4, \sigma}$ decreasing with increasing rapidity while staying positive if $\alpha>0$ as in the third row or decreasing with increasing rapidity while becoming negative if $\alpha<0$ as in the fourth row. Both the sign change and the nonmonotonic behavior in $\omega_{4, \sigma}$, as a function of the rapidity acceptance in the center panel of Fig. 1 and even more so as a function of the rapidity bin in the right panel of Fig. 1 are new results of this work. They arise from the rapidity dependence of $\mu_{B}$ at freeze-out in collisions at RHIC BES energies, and provide distinctive signatures if decreasing the beam energy in this scan takes $\mu_{B, 0}$ past a critical point.

To complement Fig. 1, Fig. 2 shows the cumulant ratio $\omega_{4, \sigma}$ binned in rapidity for a fixed beam energy (fixed $\mu_{B, 0}$ ) as the location of the critical point $\mu_{B}^{c}$ is changed. There are several features of binning the cumulants in rapidity which we believe will make doing so an important way to probe the critical region, if a critical point is discovered in the RHIC BES. First, $\omega_{4, \sigma}$ increases with $\left|y_{c}\right|$ if freeze-out at midrapidity occurs at a $\mu_{B, 0}$ that is well below $\mu_{B}^{c}$, in the red region, whereas it will decrease with $\left|y_{c}\right|$ if $\mu_{B, 0}$ is closer to or larger than $\mu_{B}^{c}$, in the blue region. This remains true even if $\alpha$ changes sign, as demonstrated in the bottom row of Fig. 1. Furthermore, a sign change in $\omega_{4, \sigma}$ as a function of $y$ will be easier to see upon binning in $\left|y_{c}\right|$ since not doing so, as in the middle panels, can obscure it by mixing data from different regions in rapidity. 

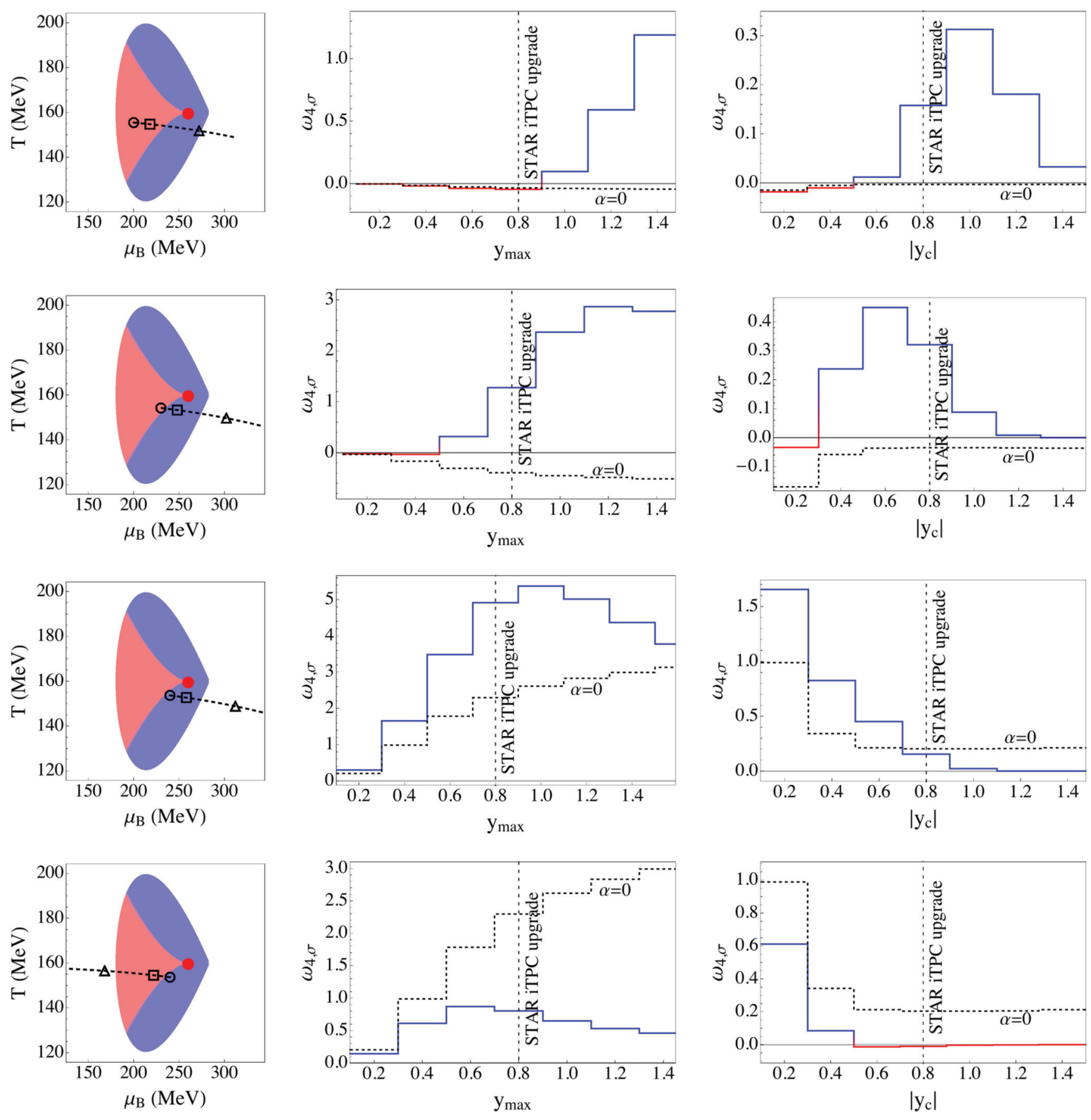

FIG. 1. In the left column we see that we have assumed the existence of a critical point (red dot) at $\left(\mu_{B}^{c}, T\right)=(260,160) \mathrm{MeV}$ whose critical region, bounded by the contour where $\xi=1 \mathrm{fm}$, is colored red and blue. The colors denote the sign of $\omega_{4, \sigma}$, with $\omega_{4, \sigma}>0$ in blue and $\omega_{4, \sigma}<0$ in red. Different rows correspond to different assumptions for where on the phase diagram a heavy-ion collision freezes out, cf. collisions with varying beam energy. The black circles show where freeze-out occurs at midrapidity, from top to bottom with $\mu_{B, 0}=200,230$, 240 , and $240 \mathrm{MeV}$. The black dashed curves show how the freeze-out conditions change with increasing space-time rapidity, with the circle, square, and triangle indicating freeze-out at $y_{s}=0,0.6$, and 1.2, respectively. In the top three rows, we have chosen $\alpha=50 \mathrm{MeV}$ [see Eq. (1)] while for the bottom row we have chosen $\alpha=-50 \mathrm{MeV}$. The middle column shows how $\omega_{4, \sigma}$ computed for a rapidity acceptance $|y|<y_{\text {max }}$ depends on $y_{\max }$. The right column shows how $\omega_{4, \sigma}$ computed in a pair of bins with width $\Delta y=0.4$ centered at $\pm y_{c}$ depends on $y_{c}$. The results in the middle column sum over a wide range of rapidities (with $|y|$ between 0 and $y_{\max }$ ) which freeze-out with a range of $\mu_{B}$, meaning that features from the left column are more directly visible in the right column than in the middle. In both the center and right columns, the black dotted lines show $\omega_{4, \sigma}$ with $\alpha=0$, i.e. what would have been obtained if $\mu_{B}=\mu_{B, 0}$, denoted by the black circles in the left column, everywhere. The results shown in the right and middle columns should not be taken as quantitative predictions since they depend on the many assumptions that we made for illustrative purposes; they are illustrative of qualitative features to be expected in the rapidity dependence of cumulants if steps in beam energy take us past a critical point. 


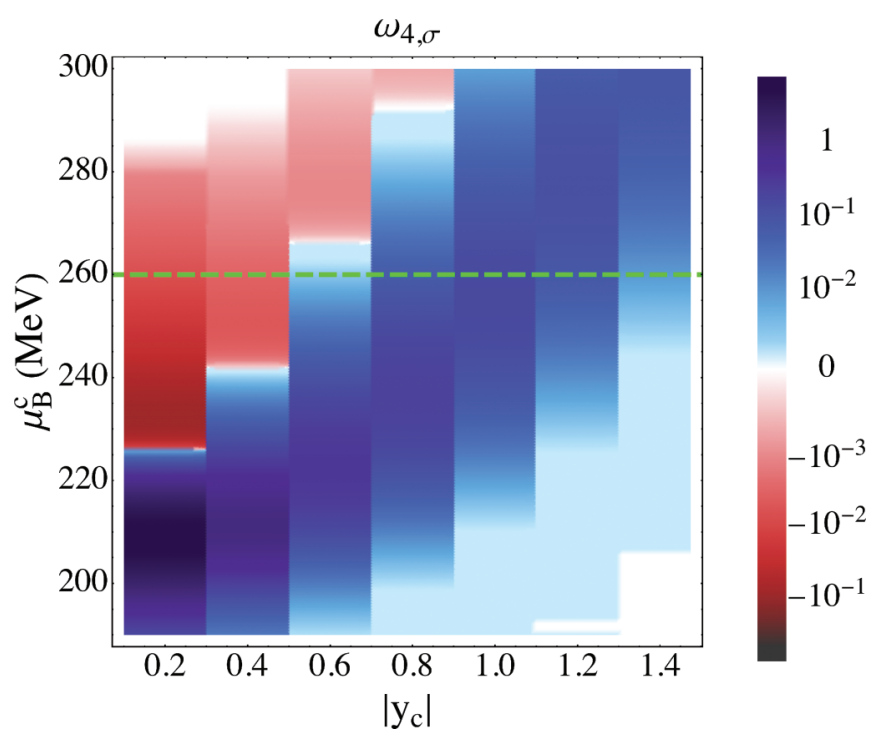

FIG. 2. Behavior of $\omega_{4, \sigma}$ when the freeze-out conditions are as in the first row of Fig. 1 with $\mu_{B, 0}=200 \mathrm{MeV}$ and $\alpha=50 \mathrm{MeV}$, but here the location of the critical point ranges from $\mu_{B}^{c}=190$ $\mathrm{MeV}$ to $\mu_{B}^{c}=300 \mathrm{MeV}$. For each value of $\mu_{B}^{c}$, namely, for each horizontal slice across the figure, color indicates the value of $\omega_{4, \sigma}$ as a function of $\left|y_{c}\right|$, with $\Delta y=0.4$ fixed as in Fig. 1. The slice indicated by the green dashed line corresponds to the top-right panel of Fig. 1. For $\mu_{B}^{c}<\mu_{B, 0}\left(\mu_{B}^{c}>\mu_{B, 0}\right), \omega_{4, \sigma}$ decreases (increases) with increasing $\left|y_{c}\right|$.

Even in cases where the sign change in $\omega_{4, \sigma}$ is visible in the middle column, as in the first and second rows, it happens at a lower rapidity in the right panel than in the middle panel, making it more feasible to observe at STAR via binning in $\left|y_{c}\right|$.

We conclude that the rapidity dependence of $\mu_{B}$ at RHIC energies may result in qualitative signatures of critical fluctuations manifest in the rapidity dependence of the cumulant ratio $\omega_{4, \sigma}$. Complementary to scanning the phase diagram by taking steps in beam energy, the rapidity dependence of $\mu_{B}$ provides additional scans of small regions of the phase diagram. We have seen that nonmonotonicity and a sign change of the critical contribution to $\omega_{4, \sigma}$ as a function of rapidity will arise if the BES includes energies on both sides of a critical point. Binning the cumulants in rapidity provides a sensitive probe of these effects. Signatures of critical behavior in the $\sqrt{s}$ dependence of $\omega_{4, \sigma}$ can therefore be cross-checked by looking for qualitative changes in the rapidity dependence of $\omega_{4, \sigma}$ between beam energies on either side of the critical point. We have made arbitrary choices at many points, for illustrative purposes. A future quantitative study should include investigation of changes to these choices, as well as an investigation of consequences of various relevant physical effects which we have neglected in this exploratory study. These include the consequences of the variation of the baryon density across the fireball at a given space-time rapidity originating from fluctuations in baryon stopping, for example, as in Ref. [35]. We note, however, that determining the value of $\alpha$ from experimental data as in Ref. [34] as we propose, will incorporate the most important such consequence. The value of $\alpha$ obtained in this way is an average over many events with fixed $\sqrt{s}$, meaning that it will be important in future work to assess how $\kappa_{4}$ is influenced by event-by-event fluctuations in baryon stopping over and above their effect on the value of $\alpha$. Future studies should also include an analysis of the quantitative effects of nonequilibrium dynamics, in particular critical slowing down, on the growth of the correlation length and hence on the values of $\sqrt{s}$ or $y_{c}$ at which the qualitative features that we have found occur [41,44-46]. The effects of baryon number conservation as well as fluctuations in baryon stopping on the cumulants have been studied $[47,48]$ and should be included in a quantitative model.

Acknowledgments. We are grateful to U. Heinz, J. Jia, R. Lacey, X. Luo, J.-Y. Ollitrault, H. Petersen, L. Ruan, B. Schenke, C. Shen, M. Stephanov, and R. Venugopalan for helpful conversations and Francesco Becattini for a helpful private communication about Ref. [34]. We are grateful to Wilke van der Schee for help in realizing the visualization that we have employed in Fig. 2. This work was supported by the Office of Nuclear Physics of the U.S. Department of Energy within the framework of the Beam Energy Scan Theory (BEST) Topical Collaboration and under Contracts No. DE-SC0011090 (JB, KR, YY) and No. DE-SC0012704 (BNL).
[1] Y. Akiba et al., arXiv:1502.02730.

[2] A. Aprahamian and others, Reaching for the horizon: The 2015 long range plan for nuclear science (2015), http://science.energy.gov/ $/ \mathrm{media} / \mathrm{np} / \mathrm{nsac} / \mathrm{pdf} /$ 2015LRP/2015_LRPNS_091815.pdf.

[3] W. Busza, K. Rajagopal, and W. van der Schee, Annu. Rev. Nucl. Part. Sci. 68, 339 (2018).

[4] F. Karsch, Nucl. Phys. A 698, 199 (2002).

[5] Y. Aoki, G. Endrodi, Z. Fodor, S. D. Katz, and K. K. Szabo, Nature 443, 675 (2006).

[6] S. Borsanyi, Z. Fodor, C. Hoelbling, S. D. Katz, S. Krieg, and K. K. Szabó, Phys. Lett. B 730, 99 (2014).

[7] A. Bazavov et al. (HotQCD Collaboration), Phys. Rev. D 90, 094503 (2014).
[8] T. Bhattacharya et al. (HotQCD Collaboration), Phys. Rev. Lett. 113, 082001 (2014).

[9] X. Luo and N. Xu, Nucl. Sci. Tech. 28, 112 (2017).

[10] P. de Forcrand, PoS LAT2009, 010 (2009).

[11] H.-T. Ding, F. Karsch, and S. Mukherjee, Int. J. Mod. Phys. E 24, 1530007 (2015).

[12] K. Rajagopal, Nucl. Phys. A 661, 150 (1999).

[13] K. Rajagopal and F. Wilczek, in At the Frontier of Particle Physics. Handbook of QCD, Vol. 3, edited by M. Shifman and B. Ioffe (World Scientific, Singapore, 2000), pp. 2061-2151.

[14] M. A. Stephanov, Prog. Theor. Phys. Suppl. 153, 139 (2004).

[15] M. A. Stephanov, PoS LAT2006, 024 (2006). 
[16] I. G. Bearden et al. (BRAHMS Collaboration), Phys. Rev. Lett. 93, 102301 (2004).

[17] J. Cleymans, H. Oeschler, K. Redlich, and S. Wheaton, Phys. Rev. C 73, 034905 (2006).

[18] A. Andronic, P. Braun-Munzinger, K. Redlich, and J. Stachel, arXiv: 1710.09425.

[19] N. S. Geraksiev et al. (MPD Collaboration), J. Phys.: Conf. Ser. 1023, 012030 (2018).

[20] T. Ablyazimov et al. (CBM Collaboration), Eur. Phys. J. A 53, 60 (2017).

[21] L. Adamczyk et al. (STAR Collaboration), Phys. Rev. Lett. 112, 032302 (2014).

[22] X. Luo (STAR Collaboration), PoS CPOD2014, 019 (2015).

[23] X. Luo, Nucl. Phys. A 956, 75 (2016).

[24] Y. Wang (STAR Collaboration), J. Phys.: Conf. Ser. 535, 012022 (2014).

[25] M. A. Stephanov, Phys. Rev. Lett. 102, 032301 (2009).

[26] Y. Hatta and M. A. Stephanov, Phys. Rev. Lett. 91, 102003 (2003); 91, 129901(E) (2003).

[27] C. Athanasiou, K. Rajagopal, and M. Stephanov, Phys. Rev. D 82, 074008 (2010).

[28] M. A. Stephanov, Phys. Rev. Lett. 107, 052301 (2011).

[29] M. A. Stephanov, J. Phys. G 38, 124147 (2011).

[30] J. Steinheimer, M. Bleicher, H. Petersen, S. Schramm, H. Stocker, and D. Zschiesche, Phys. Rev. C 77, 034901 (2008).

[31] B. Ling and M. A. Stephanov, Phys. Rev. C 93, 034915 (2016).

[32] A. Bzdak, V. Koch, and N. Strodthoff, Phys. Rev. C 95, 054906 (2017).
[33] M. Li and J. I. Kapusta, Phys. Rev. C 95, 011901 (2017).

[34] F. Becattini, J. Cleymans, and J. Strumpfer, PoS CPOD07, 012 (2007).

[35] C. Shen and B. Schenke, Phys. Rev. C 97, 024907 (2018).

[36] K. Rajagopal and F. Wilczek, Nucl. Phys. B 399, 395 (1993).

[37] J. Berges and K. Rajagopal, Nucl. Phys. B 538, 215 (1999).

[38] M. A. Halasz, A. D. Jackson, R. E. Shrock, M. A. Stephanov, and J. J. M. Verbaarschot, Phys. Rev. D 58, 096007 (1998).

[39] M. A. Stephanov, K. Rajagopal, and E. V. Shuryak, Phys. Rev. Lett. 81, 4816 (1998).

[40] M. A. Stephanov, K. Rajagopal, and E. V. Shuryak, Phys. Rev. D 60, 114028 (1999).

[41] B. Berdnikov and K. Rajagopal, Phys. Rev. D 61, 105017 (2000).

[42] J. Zinn-Justin, Quantum Field Theory and Critical Phenomena (Clarendon, Oxford, 1996).

[43] P. Parotto, PoS CPOD2017, 036 (2018).

[44] S. Mukherjee, R. Venugopalan, and Y. Yin, Phys. Rev. C 92, 034912 (2015).

[45] S. Mukherjee, R. Venugopalan, and Y. Yin, Phys. Rev. Lett. 117, 222301 (2016).

[46] M. Sakaida, M. Asakawa, H. Fujii, and M. Kitazawa, Phys. Rev. C 95, 064905 (2017).

[47] A. Bzdak, V. Koch, and V. Skokov, Eur. Phys. J. C 77, 288 (2017).

[48] A. Bzdak and V. Koch, Phys. Rev. C 96, 054905 (2017). 